\title{
KNOWLEDGE AND MALPRACTICES IN PEDIATRICS DIARRHEA MANAGEMENT BY IRAQI MOTHERS
}

\section{SAMER IMAD MOHAMMED ${ }^{*}$, AYA THAER SABRY², DANIA THAER SABRY², BOROOJ HASSAN MOHAMMED ${ }^{3}$}

${ }^{1}$ Department of Clinical Pharmacy, Faculty Member, College of Pharmacy,University of Baghdad, Baghdad, Iraq. ${ }^{2}$ Department of Clinical Pharmacy, College of Pharmacy,University of Baghdad, Baghdad, Iraq. ${ }^{3}$ Department of Clinical Pharmacy, College of pharmacy,Univeristy of Baghdad ,Baghdad,Iraq.

Email: samer.jameel@copharm.uobaghdad.edu.iq

Received: 22 May 2018, Revised and Accepted: 21 June 2018

ABSTRACT

Objectives: In developing countries like Iraq, diarrhea was responsible for $70 \%$ of deaths among pediatrics. This study was designed to determine Iraqi mothers' knowledge and malpractices associated with diarrhea management in pediatrics.

Methods: A cross-sectional pilot study was done on a convenient sample of mothers in Baghdad - Iraq. Data collection was done using a validated questionnaire specifically designed for this study.

Result: Most participants preferred to consult physicians or pharmacists about pediatrics diarrhea management. Breastfeeding was stopped by $19 \%$ of participants, whereas $35 \%$ of mothers who depend on formulated milk discontinued it. Only $30 \%$ of participants use oral rehydration solution therapy always as a part of treatment. Although $54 \%$ of participated mothers certainly not used antibiotics for the diarrhea management before doctor consultation, however $52.5 \%$ of these mothers used antimotility suspensions without any consultation. $17 \%$ of participants used some herbal preparations in addition to $11 \%$ used some forms of traditional harmful preparations (Al-Sagwa) to manage diarrhea.

Conclusions: This study indicates a good level of knowledge about diarrhea management for most participants, although there are some wrong concepts which followed by a small percentage of mothers, especially the most dangerous malpractices of using Al-Sagwa.

Keywords: Knowledge, Malpractices, Pediatrics, Infants, Diarrhea, Al-Sagwa.

(C) 2018 The Authors. Published by Innovare Academic Sciences Pvt Ltd. This is an open access article under the CC BY license (http://creativecommons. org/licenses/by/4. 0/) DOI: http://dx.doi.org/10.22159/ajpcr.2018.v11i8.27454

\section{INTRODUCTION}

Diarrhea and its complications remain a major cause of morbidity and mortality in children, especially in the developing countries. It is the second most common cause of death in children under 5 years of age worldwide and is responsible for 2.4 million deaths annually [1]. In Iraq, diarrhea is responsible for $70 \%$ of deaths among children, and every year each child suffers from an average of 6 episodes of diarrhea [2]. Parasitic diarrhea is one of the most prevalent types among the children in Iraq with an estimated prevalence rate of $22 \%$ [3], unlike other diseases, diarrhea is generally not considered as an illness, and thus most diarrheal cases are either not managed at all or managed at home through traditional approaches. About onehalf of children under 5 years did not receive any health-care support and about one-third of them did not receive any treatment at all [4]. Mothers are the key caregivers in treating diarrheal episodes at home. They took the responsibility of decision about the type of food given to the child and the overall management of the disease. Therefore, mothers' knowledge and attitude about the cause of diarrhea is critically important in taking appropriate timely actions [5]. Many factors can influence mothers' knowledge about management of childhood diarrhea, such as educational status, prior experience of managing the disease, and even ethnicity [6]. In addition, factors such as mothers' occupation, husbands' employment status, family income, and family size are linked with mothers' knowledge about diarrhea and its management besides mothers' personal attitude and behavior [7]. Although several studies have been designed to evaluate the attitude of diarrhea management in pediatrics throughout the world, there are no previous studies in Iraq. Thus, this study was designed to determine mothers' degree of knowledge and associated malpractices regarding pediatric diarrhea management in Baghdad community, Iraq.

\section{METHODS}

A pilot cross-sectional study was performed on a convenience sample of mothers in Baghdad, Iraq from November 2017 to April 2018. Data were collected using a questionnaire specifically designed for this study. The questionnaire was developed by the main author of the present study based on a literature review for closely related articles, with minor modifications on some questions to be compatible with sociodemographic nature of the Iraqi community. The questionnaire consists of two parts; the first part involves the determination of the demographic data (e.g., age, educational level, occupation, and number of children) for each participated mother. The second part consisted of 11 questions that intended to measure the knowledge and attitude of the Iraqi mothers toward diarrhea treatment. In addition, we intended to asses some involved malpractices in this regard, such as using antibiotics without indications or utilizing the most harmful approach termed (Al-Sagwa), which is a harmful traditional method that include the use of a strange mixture of many substances obtained from a dead animal (hedgehog), mixed with other poisonous substances such as lead, usually given by the witchdoctors to the infant in order to stop diarrhea. First, the questionnaire was validated by the local Scientific and Ethical Committee in the College of Pharmacy, Baghdad University. Then, the validated questionnaire was distributed among 20 mothers to test its reliability. Cronbach's alpha was 0.768 , which indicates a consistent reliability of the questionnaire. Finally, test and retest reliability was performed using Pearson's correlation and revealed significant positive correlations for the 10 questions. The correlation coefficient ( $\mathrm{r}$ ) value range was $0.63-1.0$ for all the involved items and indicates that all items provide consistent scores. Verbal consent was obtained from all participants included in the study. The authors informed the participants about the purpose of the study at the beginning of each interview. Meanwhile, the respondents were 
informed that their participation was voluntary and they were allowed to withdraw themselves at any point of time during the interview.

\section{Statistical analysis}

Statistical analysis was performed using the Statistical Package for the Social Sciences software version 16 (SPSS v. 16). Discrete variables are presented as numbers and frequencies. The Pearson correlation coefficient was used to assess the correlation between test-retest validation and for correlation between some variables.

\section{RESULTS}

The questionnaire was administered to 200 mothers in Baghdad. The demographic data are presented in Table 1.

In the present study, mother's attitude toward the management of childhood diarrheal episodes was assessed. Fig. 1 revealed that $62.5 \%$ of the participants preferred to consult their doctors about the most appropriate management, while $26.5 \%$ preferred pharmacist advice and consultation, and only $11 \%$ of them were practiced traditional home approaches.

Breastfeeding during diarrheal episodes was also an important part of the present study. The results of the present study showed that $81 \%$ of the enrolled mothers, who breastfed their infants naturally would actually continue breastfeeding, whereas 19\% of them would rather suspend breastfeeding (Fig. 2). Moreover, among the participants who fed their infants formulated or processed milk, the majority (66\%) of them keep feeding as usual and only $34 \%$ discontinued bottle-feeding (Fig. 2)

Regarding the approach of diluting the milk, the present study revealed that $66 \%$ of the mothers who use processed milk maintained feeding their infants the usual type of the processed milk without any dilution,

Table 1: Demographic data for participating mothers

\begin{tabular}{lc}
\hline Category & n (\%) \\
\hline Age of participants & \\
20-year-old & $26(13)$ \\
20-40 year-old & $136(68)$ \\
>40-year-old & $38(19)$ \\
Education & $42(21)$ \\
Primary school & $64(32)$ \\
High school & $94(47)$ \\
Bachelor degree & \\
Number of children & $75(37.5)$ \\
One child & $125(62.5)$ \\
More than one child & \\
Working status & $45(22.5)$ \\
Employed & $155(77.5)$ \\
Housewife & \\
\hline
\end{tabular}

All values are expressed as numbers and percentages; $n$ : Number of subjects

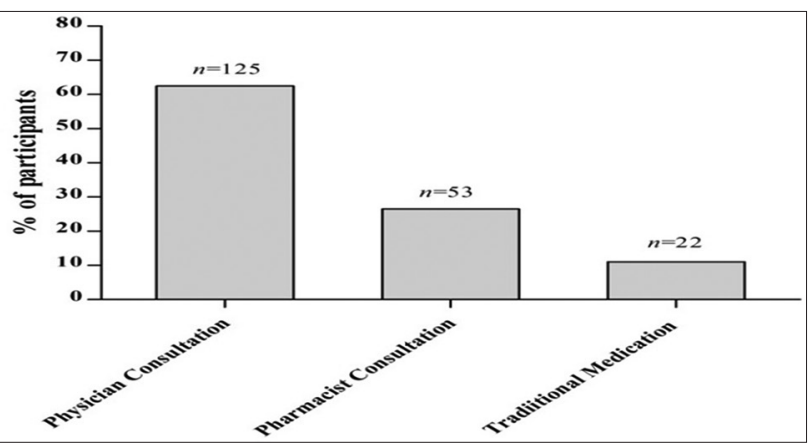

Fig. 1: Distribution of participants $(\%)(n=200)$ based on their attitudes toward diarrhea treatment whereas $44 \%$ of them diluted the formula with water before feeding their infants during the episodes of diarrhea (Fig. 3). Meanwhile, it was also reported that $59 \%$ of the mothers who fed their infants a processed milk formula changed the type of the processed milk to some other forms that were particularly formulated to be given during diarrheal episodes (Fig. 3).

The use of oral rehydration solution (ORS) to prevent dehydration during diarrheal episodes was interpreted in the present study. About $30 \%$ of the participants claimed that ORS administration is important and they use it always as a part of treatment, $40 \%$ of them use ORS therapy occasionally, and the last group (30\%) never used such approach for the management of infants' diarrhea (Fig. 4).

Concerning the use of zinc-containing supplements during infants' diarrheal episodes, it was found that only $22 \%$ of mothers were administered zinc supplements as a complementary part of the treatment (Fig. 4).

Regarding the practice of using antibiotics, the present study showed that $54 \%$ of the enrolled mothers never used antibacterial drugs during treatment of diarrheal episodes without physician consultation; however, $33.5 \%$ of mothers visited the nearby community pharmacy and ask for the pharmacist advice, whereas $12.5 \%$ of them would reuse the antibacterial agents already available at home for treatment (Fig. 5). The use of antimotility suspensions available in the pharmacies in order to stop or reduce diarrheal episodes was also assessed. About $52.5 \%$ of mothers used these medications and $47.5 \%$ of them never used such therapies for childhood diarrheal management (Fig. 5).

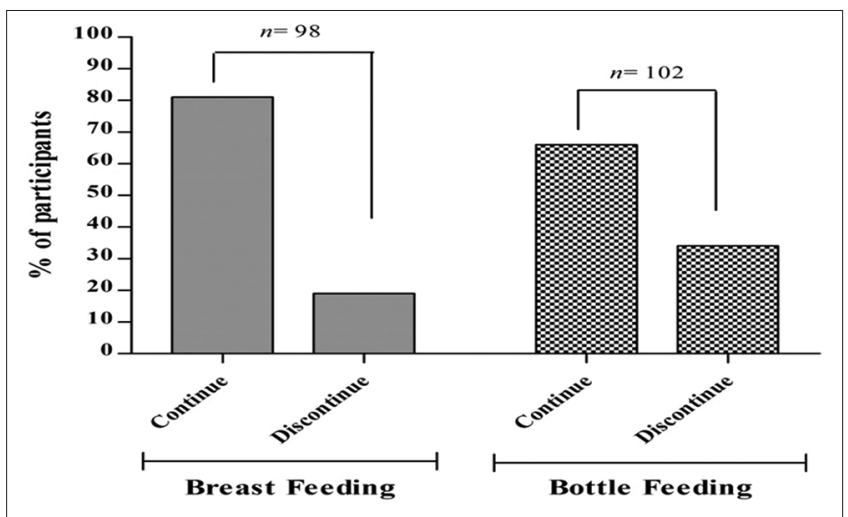

Fig. 2: Participants distribution $(\%)(n=200)$ based on the practice of continuing discontinuing bottle- and breastfeeding of their children during diarial episodes

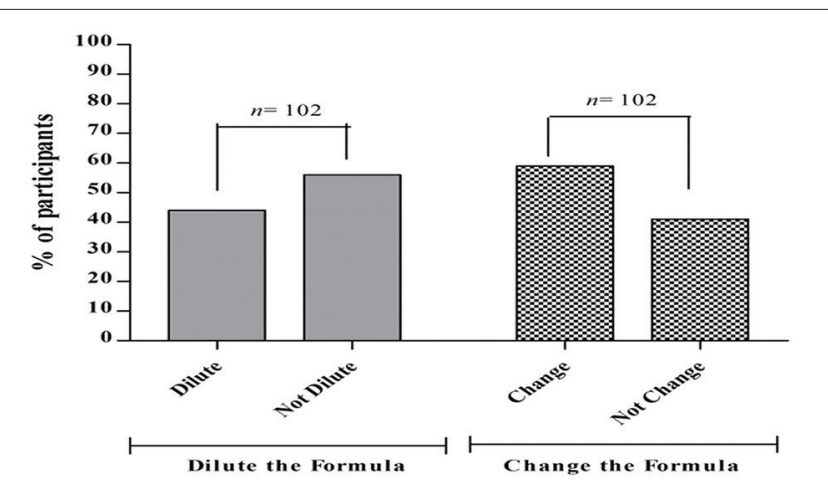

Fig. 3: The mothers' practice of diluting or changing the processed milk formula for their bottle-fed children during diarrheal episodes 
Common ailments for which participants preferred to self-medicate their children were noted. These included some herbal remedies and AlSagwa. It was found that $17 \%$ of them used some herbal preparations as a therapy for diarrhea, whereas only $11 \%$ of participants used Al-Sagwa (Fig. 6).

In Table 2, the data demonstrated that there is no significant correlation between some participants' related age, level of education, and the number of children and attitude toward some malpractices during management of diarrheal episodes in children.

\section{DISCUSSION}

Diarrhea is a worldwide health issue associated with various beliefs and practices to manage it across the globe. Several factors might contribute to the beliefs and practices, which might vary according to region, country, ethnicity, culture, and geographical location [6]. The enrolled mothers in this study were of the age group between 20 and 40 years. Most mothers in this age group are considered to be more enthusiastic and highly interested in getting knowledge about their child's health.' This result was similar to the result observed in a previous study performed to assess the awareness and attitude toward diarrhea among mothers of children below 5 years where most of participants $(62.60 \%)$ were in similar age group [8]. The majority of contributors are well educated, and this can be attributed to the fact that educated women are more interested in improving their knowledge about preventative measures that could decrease the impact of diarrhea on their children. It is well known that mortality can be decreased by half if appropriate preventative measures are followed by mothers [9]. Diarrheal episodes are more common in the lower educated group and low socioeconomic status families with a prevalence of overcrowding [10]. The present

Table 2: Correlation between the age, level of education, and number of children of the participants and some malpractices such as herbal use, Sagwa use, and use of antidiarrheal suspensions

\begin{tabular}{lccc}
\hline Category & $\begin{array}{c}\text { Herbal } \\
\text { Formula }\end{array}$ & Al-Sagwa & $\begin{array}{c}\text { Antidiarrheal } \\
\text { drugs }\end{array}$ \\
\hline $\begin{array}{l}\text { Age } \\
\text { Correlation } \\
\text { coefficient }\end{array}$ & 0.010 & 0.077 & 0.063 \\
$\begin{array}{l}\text { Sig. (2-tailed) } \\
\text { Education }\end{array}$ & 0.890 & 0.281 & 0.372 \\
$\begin{array}{l}\text { Correlation } \\
\text { coefficient }\end{array}$ & 0.017 & 0.101 & 0.079 \\
$\begin{array}{c}\text { Sig. (2-tailed) } \\
\text { No. of children } \\
\text { Correlation } \\
\begin{array}{l}\text { coefficient } \\
\text { Sig. (2-tailed) }\end{array}\end{array}$ & 0.806 & 0.155 & 0.267 \\
\hline
\end{tabular}

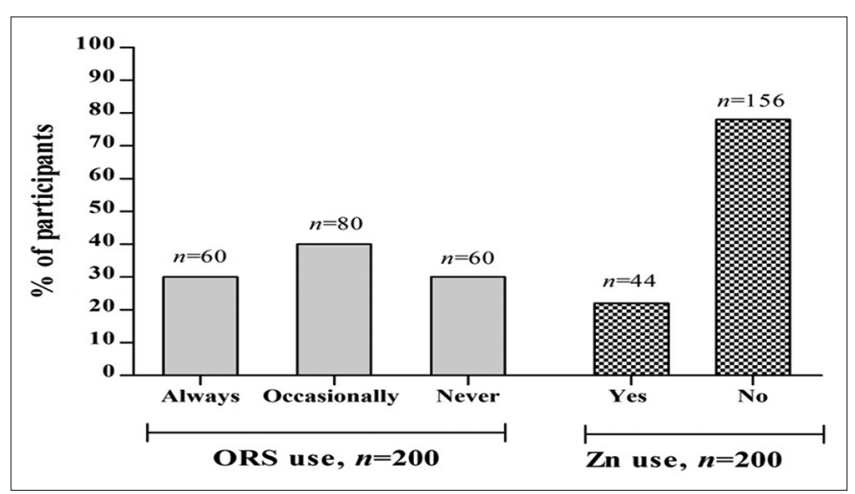

Fig. 4: The use of oral rehydration solution therapy and zinc supplement during diarrheal episodes; $n$ : Number of subjects study showed that most of the participants preferred consulting a physician or a pharmacist to treat diarrhea; this can be attributed to the fact that most of the mothers enrolled in this study are well educated, so they prefer to get appropriate treatment for their child. Only $11 \%$ of them use home medications without consultation, this is in opposition to other studies performed in Mali [11] and Vietnam[ [12], which showed that treatment of diarrhea begins mostly in the home with the use of traditional medicines and/or antibiotics without consultation. The WHO and UNICEF are emphasizing the crucial role of breastfeeding as a significant protective factor against morbidity and mortality related to diarrhea [13]. In all diarrheal cases, breastfeeding must be continued, in fact, it should be increased to the greatest extent as possible. The major benefit from this is that breastfed infants have fewer episodes of severe diarrhea and a lower risk of dehydration than infants who are not being breastfed [14]. One of the wrong concepts that followed by many mothers is stopping breastfeeding during diarrheal episodes as they assumed that breast milk will aggravate the condition. This wrong concept indicated also in a study of mothers' beliefs and barriers about childhood diarrhea in Nepal, where many participants believed that breast milk sometimes considered harmful [15]. The present study showed that only $19 \%$ of enrolled mothers suspended breastfeeding, while the majority of them $(81 \%)$ continued breastfed their children during the diarrheal episodes. This result was found to be in tune with that reported by McLennan which indicated that only a few (3\%) of the mothers believed that breastfeeding should be postponed [16]. Although only $<20 \%$ of mothers in this study believed that stopping breastfeeding is better for the child with diarrhea, this may be related to the convenient sample of this study where most of the participants are well educated. This requires increasing the awareness of mothers about the importance of continuing breastfeeding during diarrheal episodes. In addition, this study showed that $66 \%$ of participants continued bottlefeeding as usual compared to $34 \%$ who discontinued such practice during their infant's diarrheal episodes. It was reported that $11 \%$ of caregivers in Mirzapur, Bangladesh curtailed formulated milk during their infant's diarrheal episodes [17], while the percentage reported in

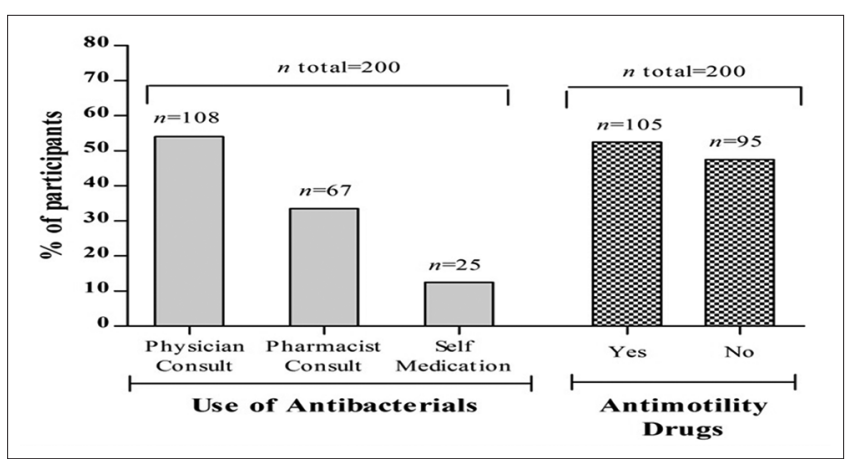

Fig. 5: The mothers' attitude of using antibiotics and antimotility agents during diarrheal episodes

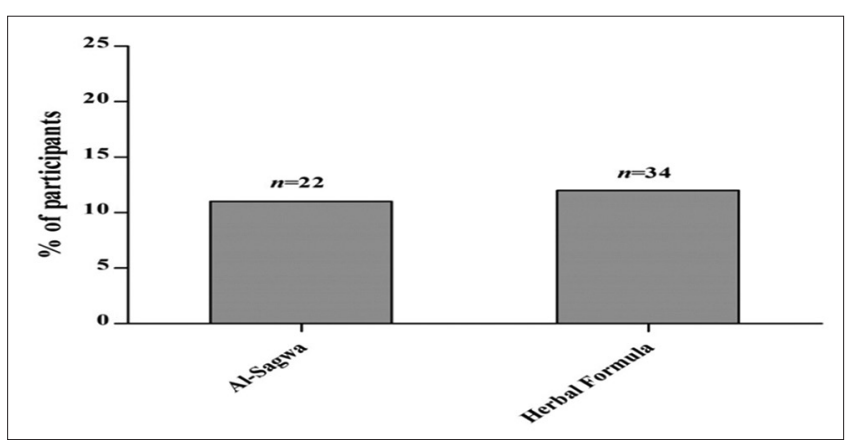

Fig. 6: The attitude of mothers $(\% ; n=200)$ toward the use of either Al-Sagwa or a herbal formula for the management of diarrheal episodes of their children 
Kenya's Nyanza province was much higher, approximated as $80 \%$ of the caregivers [18]. Suspending bottle-feeding was also reported in previous studies performed in Saudi Arabia [19] and Egypt [20]. Meanwhile, the malpractice of milk dilution with water before bottle-feeding of infants during diarrheal episodes is a common misunderstanding, since this would probably cutoff some of the nutritional value, not to mention the interference with the normal growth and development of the child. About $44 \%$ of mothers were reported to do so [14].

In children younger than 6 months of age, the lack of suitable studies must lead to caution and use of specific lactose-free or extensively hydrolysate formulae, especially in case of severe and/or prolonged diarrhea [21]. In this study, we observed that only $41 \%$ of the mothers maintained feeding their infants with the same milk formula, whereas the majority of them switched feeding to the use of lactose-free form.

Although the crucial role of ORS has been discussed in previous studies, until now many mothers did not use or ignore the use of ORS. This was clearly demonstrated in the present study, where only $30 \%$ of mothers used ORS during their child diarrhea, which came in line with other studies $[8,22]$ This might be related to many factors corresponding to the poor compliance with instructions for use in addition to the appropriate method of administration. In fact, the uncommon use of ORS by the mothers appears to be a major one, which can be attributed to the poor knowledge about the role of this approach in the management of infants' diarrhea. Currently, many health institutions attempted to raise the parents' awareness about such approach through advertisement.

Antimicrobials are suggested only for the management of bloody diarrhea or suspected cholera with severe dehydration [14]. The overuse of such antibiotics might delay the visit to the health-care center to seek medical intervention; in addition, the family will spend too much money and waste their financial resources [23]. The present study showed a misuse of antibiotics to manage diarrhea in children by $12.5 \%$ of the participants, which was in conflict with the higher misuse of antibiotics reported by others, which mentioned $74 \%$ in Egypt [24] or 73\% of mothers in the Dominican Republic [16]. This may be linked to a well-educated convenient sample of participants in this study, in addition to the effective role of Iraqi heath government institution in the last few years, which directed toward the precise use of antibiotics in Iraq. In contrary, more than $52.5 \%$ of the participants in this study widely used the antimotility medications to treat childhood diarrhea, which is not recommended according to the WHO guideline that indicates the first-line management of diarrhea in children below 5 years of age with continued feeding, increased fluids, and supplemental zinc for 10-14 days to prevent dehydration [14].

This result was not in tune with many previous studies; the first one performed in Egypt while the other study in a rural district of Vietnam [24], which reported that the use of antidiarrheal and antimotility agents was generally lower than the reported use of antibiotics [12].

Herbal preparations are misused by $17 \%$ of the participants, which seem to be lower than that reported by a previous study performed in the Gambia, where the herbal preparations misused by $18.7 \%$ of the participants [25]. On the other hand, it was higher than that reported in a study performed in Ethiopia, which indicated that only $0.5 \%$ of caregivers utilized traditional herbs to treat pediatrics diarrhea [26].

In Iraq, there is a wrong traditional method, the mothers self-practiced to treat diarrhea and some other problems in newborns, which include the use of what is known as "Al-Sagwa;" it is a strange formula consisted of a mixture of parts of a dead animal (hedgehog) mixed with other poisonous substances, such as lead, usually recommended by the witchdoctors if the newborn is suffering from diarrhea. However, this formula causes many serious complications such as kidney failure, bacterial poisoning, intestinal bleeding, in addition to the increase of salts retention in the body, and might lead to death even after hospital admission and be performing all necessary medical measures.
The present study showed that only $11 \%$ of the participants used this dangerous poisonous mixture to manage their children's diarrhea. This percentage might be higher if the study included a rural area in Iraq, as many mothers are illiterate in addition to the absence of a nearby medical center or difficulty to reach these centers. All of these factors encourage mothers to go to witchdoctors and use Al-Sagwa.

Several trials were proven the therapeutic efficacy of zinc by resolution of small bowel damage and shortening duration of diarrhea [27]. It was found that zinc can actually increase water and electrolytes absorption, enhance the intestinal epithelial cells to regenerate, raise brush border enzymes levels, and improve the immune response in order to clear the intestine from any remaining pathogens. Zinc can also enhance the host resistance against pathogens in order to reduce the severity and duration of the diarrheal episodes [28]. The WHO and UNICEF recommended that children $<6$ months must take $10 \mathrm{mg}$ of zinc supplements, whereas children $>6$ months can take $20 \mathrm{mg}$ of such supplements as universal treatment [1]. Furthermore, a combination of zinc and Vitamin A appears to be more effective than either Vitamin A or zinc alone in reducing prolonged diarrhea [29]. The present study showed less than a quarter of the participants used zinc for their child's diarrhea. Similarly, a study conducted by Rokkapanaver et al. reported much lower awareness level, where the percentage of zinc administration by mothers was only $3.8 \%$ after medical consultation. The reported non-compliance is the result of the mothers' poor knowledge about the importance of zinc in treating diarrheal episodes, even when recommended by the pediatrician. Only $17 \%$ of the participants in the present study actually committed to the use of zinc and appreciate the beneficial effects from its use, whereas $29 \%$ could not tell what the role of zinc supplements in diarrheal management is. This indicates the need to highlights the role of health advertisements and education to make mothers more familiar with the zinc-based therapy since mothers represent the home health caregivers [30]. The study clearly showed that there is no correlation between some wrong concepts followed to treat diarrhea and some factors such as age, level of education, or the prior experience obtained from previous children; this indicates a low level of awareness by all mothers without regarding these factors.

\section{CONCLUSION}

This study indicates a good level of knowledge about the management of diarrhea in most of the participants although there are some wrong concepts followed by a minority of mothers, especially the most dangerous practice of using Al-Sagwa to treat infants' diarrhea that may lead to death. The study focuses on the importance of raising the awareness level by advertising about the accurate methods of management and correct use of ORS and zinc in addition to avoiding any non-prescribed antibiotics and antidiarrheal medications.

\section{ACKNOWLEDGMENT}

The authors would like to thank Professor Dr. Saad A. Hussain, Senior Lecturer, Dr. Ali Aziz, and Senior lecturer Dr. Ehab Mudher Mikhael for support and comments that greatly improved the manuscript.

\section{AUTHOR'S CONTRIBUTION}

The four authors are participated in collecting the data and writing the articles.

\section{ETHICAL STATEMENT}

The study and the questionnaire were validated by the local Scientific and Ethical Committee in College of Pharmacy, Baghdad University. Verbal consent was obtained from all participants included in the study. The authors informed the participants about the purpose of the study at the beginning of each interview. Meanwhile, the respondents were informed that their participation was voluntary and they were allowed to withdraw themselves at any point of time during the interview. 


\section{FUNDING SUPPORT DISCLOSURE STATEMENTS}

No funding support received for this study. The authors themselves will support any publication fees.

\section{CONFLICT OF INTEREST}

Not present for all authors.

\section{REFERENCES}

1. Cooke M. Causes and management of diarrhea in children in a clinical setting. S Afr J Clin Nutr 2010;23:140-7.

2. Ala'din A. Health in Iraq: The Current Situation, our Vision for the Future and Areas of Work. Baghdad: Ministry of Health; 2004.

3. Waqar A, Hassanain A, Alyaa A. Intestinal parasitic diarrhea among children in Baghdad-Iraq. Trop Biomed 2014;31:499-506.

4. Mukhtar A, Izham MM, Pathiyil R. Mothers' knowledge, attitude and practice regarding diarrhea and its management in Morang Nepal: An interventional study. Trop J Pharm Res 2012;11:847-54

5. Nigatu M, Tadesse A. Knowledge, perception, and management skills of mothers with under-five children about diarrhoeal disease in indigenous and resettlement communities in Assosa District, Western Ethiopia. J Health Popul Nutr 2015;33:20-30.

6. Anidi I, Bazargan M, James F. Knowledge and management of diarrhea among underserved minority parents/caregivers. Ambul Pediatr 2002;2:201-6.

7. Mukhtar A, Mohamed Izham M, Pathiyil R. A survey of mothers' knowledge about childhood diarrhoea and its management among a marginalised community of Morang, Nepal. Australas Med J 2011;4:474-9.

8. Gondar NR, Farooq D. Diarrhea, nutrition and oral rehydration therapy: Awareness, attitude and practices among mothers of children under five years. Int J Contemp Pediatr 2017;4:1995-2001.

9. Available from: https://www.data.unicef.org/topic/child-health/diarrhoealdisease. [Last accessed on 2017 Aug 03].

10. Padhy S, Sethi R, Behera N. Mother's knowledge, attitude and practice regarding prevention and management of diarrhea in children in Southern Odisha. Int J Contemp Pediatr 2017;4:966-71.

11. Ellis AA, Winch $\mathrm{P}$, Daou Z, Gilroy KE, Swedberg E. Home management of childhood diarrhoea in southern Mali--implications for the introduction of zinc treatment. Soc Sci Med 2007;64:701-12.

12. Le TH, Ottosson E, Nguyen TK, Kim BG, Allebeck P. Drug use and self-medication among children with respiratory illness or diarrhea in a rural district in Vietnam: A qualitative study. J Multidiscip Healthc 2011:4:329-36.

13. Kuhn L, Aldrovandi G. Survival and health benefits of breastfeeding versus artificial feeding in infants of HIV-infected women: Developing versus developed world. Clin Perinatol 2010;37:843-62.

14. World Health Organization. UNICEF, The Treatment of Diarrhea. Geneva: WHO; 2005. p. 1-5.
15. Ansari M, Ibrahim MI, Hassali MA, Shankar PR, Koirala A, Thapa NJ. Mothers' beliefs and barriers about childhood diarrhea and its management in Morang district, Nepal. BMC Res Notes 2012;5:576.

16. McLennan JD. Home management of childhood diarrhoea in a poor periurban community in Dominican Republic. J Health Popul Nutr 2002;20:245-54.

17. Othero DM, Orago AS, Groenewegen T, Kaseje DO, Otengah PA. Home management of diarrhea among underfives in a rural community in Kenya: Household perceptions and practices. East Afr J Public Health 2008;5:142-6.

18. Kaatano GM, Muro AI, Medard M. Caretaker's perceptions, attitudes and practices regarding childhood febrile illness and diarrhoeal diseases among riparian communities of Lake Victoria, Tanzania. Tanzan Health Res Bull 2006;8:155-61.

19. Bella H, Ai-Freihi H, El-Mousan M, Danso KT, Sohaibani M, Khazindar MS. Knowledge, Attitudes and Practices related to Diarrhoea in Eastern Province, Saudi Arabia. J Family Community Med 1994;1:40-4.

20. Langsten R, Hill K. Diarrheal disease, oral rehydration, and childhood mortality in rural Egypt. J Trop Pediatr 1994;40:272-8.

21. Chouraqui J, Michard-Lenoir A. Feeding infants and young children with acute diarrhea. Arch Pediatr Organe Off Soc Fr Pediatr 2007; 14:176-80.

22. Buch N, Hassan M, Bhat IA. Parental awareness and practices in acute diarrhea. Indian Pediatr 1995;32:76-9.

23. Shah M, Kathiiko C, Wada A, Odoyo E, Bundi M, Miringu G, et al. Prevalence, seasonal variation, and antibiotic resistance pattern of enteric bacterial pathogens among hospitalized diarrheic children in suburban regions of central Kenya. Trop Med Health 2016;44:39.

24. El-Gilany A, Hammad S. Epidemiology of diarrheal diseases among children under age 5 years in Dakahlia, Egypt. Eastern Mediterr Health J 2005; 11:762-75

25. Sillah F, Ho HJ, Chao JC. The use of oral rehydration salt in managing children under 5 y old with diarrhea in the Gambia: Knowledge, attitude, and practice. Nutrition 2013;29:1368-73.

26. Desta BK, Assimamaw NT, Ashenafi TD. Knowledge, practice, and associated factors of home-based management of diarrhea among caregivers of children attending under-five clinic in Fagita Lekoma district, awi zone, Amhara regional state, northwest Ethiopia, 2016. Nurs Res Pract 2017;2017:8084548.

27. Black R. Zinc deficiency, infectious disease and mortality in the developing world. J Nutr 2003; 133:1485-99.

28. Bajait C, Thawani V. Role of zinc in pediatric diarrhea. Indian $J$ Pharmacol 2011;43:232-5.

29. Rahman M, Vermund S, Wahed MA, Fuchs GJ, Baqui AH, Alvarez JO, et al. Simultaneous zinc and Vitamin A supplementation in Bangladeshi children: Randomized double blind controlled trial. BMJ 2001;323:314-8

30. Dipak K, Nilratan M, Dibasish P. Assessment of knowledge and practice of mothers of children under five regarding Zinc therapy in childhood diarrhea. Int J Adv Integr Med Sci 2016;1:169-72. 